\title{
LA PRÁCTICA EDUCATIVA DESDE LA PERSPECTIVA DE LOS FONDOS DE CONOCIMIENTO E IDENTIDAD
}

\author{
Educational practice based on the funds of knowledge \\ and identity approach
}

\section{Pratique pédagogique basé sur l'approche des fonds de connaissances et identité}

\author{
Moisés Esteban-Guitart y Xénia SAuBich \\ Universidad de Girona. Facultad de Educación y Psicología. Instituto de \\ Investigación Educativa. Plaça Sant Domènec, 9. 17071 Girona, España. \\ Correo-e: moises.esteban@udg.edu
}

Fecha de recepción: marzo de 2013

Fecha de aceptación: junio de 2013

Biblid [(1130-3743) 25, 2-2013, 189-211]

\section{RESUMEN}

Este artículo presenta una discusión de la propuesta conocida como marco de las continuidades-discontinuidades familia-escuela o desajuste familia-escuela para dar cuenta e intervenir sobre la experiencia educativa del alumnado procedente de minorías culturales. El objetivo principal del artículo, fundamentado en la teoría sociocultural, es describir la relación entre la vida familiar y escolar de los alumnos según el paradigma de los fondos de conocimiento. Más específicamente, sugerimos el concepto de fondos de identidad para complementar el concepto de fondos de conocimiento a través de la ilustración de dos estrategias metodológicas (círculo significativo y dibujo identitario) que permiten a los docentes documentar los fondos de identidad para establecer vínculos entre la práctica educativa escolar y las formas de vida de los alumnos. 
Palabras clave: fondos de conocimiento, fondos de identidad, enseñanza basada en la identidad, fracaso escolar, modelo del desajuste familia-escuela, estudiantes infrarrepresentados.

\section{SUMMARY}

This article presents a discussion of the proposal known as home-school continuity-discontinuity framework, designed to explain and to intervene on the educational experience of under-represented students. The main purpose of this article, grounded in sociocultural theory, is to describe the relationship between the home and school lives of students according to the funds of knowledge approach. Specifically, we suggest the concept of funds of identity in order to complement the funds of knowledge term. In doing so, we illustrate how teachers can document funds of identity to make direct links from students' lives to classroom instruction. Two qualitative strategies are presented: self-portrait and significant circle.

Key words: funds of knowledge, funds of identity, identity-based teaching, academic failure, home-school mismatch framework, under-represented students.

\section{SOMMAIRE}

Cet article présent une discussion de la proposition connue sous le nom de cadre des continuités-discontinuités des maison-école, destiné à expliquer et à intervenir sur l'expérience éducative des étudiants sous-représentés. Le but principal de cet article, fondé sur la théorie socioculturelle, est de décrire la relation entre le foyer et la vie scolaire des élèves en fonction des l'approche fonds du savoir. Plus précisément, nous proposons le concept de fonds de l'identité afin de compléter les fonds de la connaissance. Ce faisant, nous illustrons comment les enseignants peuvent documenter des fonds de l'identité de faire des liens directs de la vie des élèves à l'enseignement en classe. Deux stratégies qualitatives sont présentées : l'autoportrait et le cercle significatif.

Mots clés: fonds du savoir, fonds de l'identité, fondée sur l'identité d'enseginement, l'échec scolaire, le cadre de discordance maison-école, étudiants sous-représentés.

\section{INTRODUCCIÓN}

Al mismo tiempo que en los Estados Unidos de América las características sociodemográficas de los docentes -normalmente mujeres blancas de clase media- se mantienen estables, en los últimos años la población estudiantil se está diversificando étnica y culturalmente. Esta discontinuidad entre la cultura escolar y la cultura familiar de los alumnos provoca estragos en el rendimiento académico. Un número desproporcionadamente elevado de niños y niñas procedentes de minorías culturales, inmigrantes, pobres, obtienen peores resultados académicos en comparación con el alumnado blanco de clase media. 
Con estas palabras introducen -Rosebery, McIntyre y González (2001, 1) - un libro que recoge distintas experiencias educativas llevadas a cabo en los Estados Unidos de América (principalmente en los estados de Arizona y California) con el objetivo de mejorar el rendimiento escolar de las minorías culturales a través de la vinculación del currículum con las formas de vida de los alumnos y alumnas (McIntyre, Rosebery y González, 2001). El objetivo es crear continuidades entre la familia y la escuela para fortalecer la actividad educativa. El enfoque que subyace a dicha propuesta se conoce como paradigma de los fondos de conocimiento (Funds of Knowledge Approach en inglés).

El objetivo que se persigue en este artículo consiste en situar dicho enfoque educativo surgido a partir de la teoría histórico-cultural basada en el legado de Vygotski (Moll, 1990, 2013). Para ello, en primer lugar se introduce el llamado "paradigma de las continuidades-discontinuidades familia-escuela" (Poveda, 2001). En segundo lugar, se describe el origen y características de la teoría de los fondos de conocimiento, así como su importancia en el terreno educativo, y algunas limitaciones que han llevado a proponer la noción complementaria de "fondos de identidad" -Funds of Identity- (Esteban-Guitart, en prensa). A continuación, se ilustra la detección, con fines pedagógicos, de los "fondos de identidad" de distintos alumnos mediante dos técnicas cualitativas -el dibujo identitario y el círculo significativo-. Finalmente, se señalan, a modo de conclusión, el alcance de las ideas expuestas, su sentido y pertinencia para el desarrollo teórico y el diseño de procesos educativos, así como posibles beneficios y limitaciones del enfoque descrito.

Desde su puesta en marcha en Tucson (Arizona), el programa y enfoque educativo fondos de conocimiento se ha implementado en distintos lugares del planeta (Andrews y Yee, 2006; Hogg, 2011; Marshall y Toohey, 2010; Moll, Amanti, Neff y González, 1992; Olmedo, 1997; Zipin, 2009), obteniendo tres resultados positivos.

En primer lugar, se ha documentado una mejora en las relaciones familia y escuela basadas en lo que los autores llaman confianza-mutual trust- (González y Moll, 2002; Vélez-Ibáñez, 1983). Como veremos posteriormente, el programa modifica el tipo de relaciones tradicionales que han mantenido profesor-padre/madre/tutor. Históricamente y, de un modo general (por supuesto existen excepciones), el contacto entre ambos se ha suscitado a partir de tutorías en las que un experto, el docente, informa a la familia sobre el rendimiento de su hijo o hija. En cambio, lo que se propone en el programa fondos de conocimiento es que el docente visite los hogares de las familias para documentar los bancos de saberes, prácticas y habilidades que estas tienen. Las familias son contextos de vida y escenarios de enseñanza y aprendizaje, al igual que el contexto escolar, de modo que se trata de conocer sus fortalezas para poderlas visibilizar y utilizar pedagógicamente. A lo largo de las visitas y el conocimiento y reconocimiento mutuo entre docentes y familias, se modifican las creencias, muchas veces prejuiciosas, que puedan existir por parte de los docentes en relación a las familias inmigrantes y por parte de 
LA PRÁCTICA EDUCATIVA DESDE LA PERSPECTIVA DE LOS FONDOS DE CONOCIMIENTO E IDENTIDAD

éstas en relación a la cultura escolar. En definitiva, se tejen lazos de colaboración, cooperación, reconocimiento. Lo que se resume con la palabra confianza mutua -mutual trust- (González y Moll, 2002; Vélez-Ibáñez, 1983).

En segundo lugar, se crean unidades didácticas a partir de los fondos de conocimiento detectados, lo que promueve que los aprendizajes sean significativos (al vincular las experiencias y conocimientos previos -las formas de vida familiarescon las informaciones nuevas -lo que se hace en la escuela-). Lo que, siguiendo a Vygotski (1962), podríamos resumir con el vínculo de los conceptos científicos (derivados de la instrucción formal) con los conceptos espontáneos (derivados de la socialización en contextos informales como la familia y la comunidad) (McIntyre, Kyle y Rightmyer, 2005). El objetivo no es que la escuela haga lo mismo que la familia hace, sino que los conceptos espontáneos (o conocimientos previos) sirvan de ancla para poder apoyar y extender los contenidos de aprendizaje de la institución escolar.

Finalmente, y como resultado de lo anterior, se ha documentado una mejora del rendimiento escolar del alumnado procedente de minorías culturales. Es decir, el programa favorece los procesos de enseñanza y aprendizaje especialmente en aquellos casos en que hay una mayor discontinuidad entre la cultura escolar y la cultura familiar (González, Moll y Amanti, 2005).

Pensamos que todo ello justifica científicamente la consideración crítica del programa que realizamos a continuación. Un programa que apenas se está empezando a implementar y ensayar en el contexto del sistema educativo español (Hinojal, Masó, Turon y Esteban-Guitart, 2011; Saubich y Esteban-Guitart, 2011).

\title{
2. El PARADIGMA DE LAS CONTINUIDADES-DISCONTINUIDADES FAMILIA-ESCUELA
}

\begin{abstract}
A partir del trabajo de Heath, otros investigadores han descrito las distintas discontinuidades que pueden padecer niños de distinta procedencia cultural entre lo que conocen, hacen y saben en su casa y el mundo de la escuela. En distintos grados, estos niños pueden no saber cómo mostrar al docente lo que saben, pueden participar en actividades extrañas para ellos y se pueden encontrar que el docente les habla de una manera poco familiar y confusa. Desde el principio, el alumnado que pertenece a grupos minoritarios y es de clase trabajadora puede encontrar la escuela un sitio confuso y a veces incómodo (Rosebery, McIntyre y González, 2001, 3).
\end{abstract}

Poveda (2001) sintetiza la lógica subyacente al marco del «desajuste familiaescuela" ("paradigma de las continuidades-discontinuidades familia-escuela") a través de una serie de supuestos y consideraciones que resumimos de la siguiente manera:

a) El proceso educativo (enseñanza-aprendizaje) -y aquí añadiríamos cualquier proceso psicológico- conlleva comunicación y, por lo tanto, contacto social -léase zona de desarrollo próximo (Vygotski, 1979), andamiaje 
(Wood, Bruner y Ross, 1976), participación guiada (Rogoff, 1990)-. Para que haya contacto social debe existir intersubjetividad (Bruner, 1990), es decir, fondos de conocimiento compartido (González, Moll y Amanti, 2005). Para el aprendiz esto implica ser competente en dos dimensiones: contenidos conceptuales del aprendizaje y formato de interacción-comunicación en el que se insertan estos contenidos (Erickson, 1982).

b) De lo anterior se desprende que el lenguaje, la comunicación, la lectoescritura y cualquier artefacto psicológico se adquiere en y es constitutivo del proceso de socialización escolar, familiar y comunitario. Estos contextos tienen sus propios fondos de conocimiento, es decir, sus propios contenidos y sus modos de comunicación.

Según la diferenciación que establece Vygotski (1962) entre conceptos espontáneos y conceptos científicos, podemos afirmar que antes de la escolarización obligatoria formal los niños y niñas ya han adquirido un repertorio complejo y significativo de habilidades comunicativas y conceptos lingüísticos, los llamados conceptos espontáneos, que son las habilidades que las criaturas traen consigo a las escuelas. Sin embargo, las aulas demandan nuevos aprendizajes conceptuales y sociales, es decir, los conceptos científicos de la cultura dominante, y es aquí donde radica el problema. El alumnado procedente de la cultura dominante realiza una transición más directa entre los conocimientos familiares y los escolares (pensamiento espontáneo frente a pensamiento científico) ya que, al fin y al cabo, se comparten muchas cosas implícitas, entre ellas las etnoteorías o concepciones sobre la educación (Broker, 2003). Sin embargo, dicha transición es más indirecta entre las clases sociales más desfavorecidas procedentes de grupos minoritarios y la cultura escolar. El alumnado socializado en grupos culturales minoritarios que no comparten aspectos implícitos de la cultura escolar del grupo mayoritario está en desventaja ya que se enfrenta a una serie de situaciones comunicativas que a veces resultan extrañas y participar en ellas puede suponer una renuncia de las identidades, códigos comunicativos, prácticas de aprendizaje, valores y conductas culturales adquiridas en el contexto familiar-comunitario. En este sentido, recuperando la cita con la que iniciábamos el artículo, los alumnos alófonos pueden padecer ciertas dificultades a la hora de mostrar a sus docentes todo lo que saben, implicándose en actividades que muchas veces son extrañas y desconocidas para ellos. Desde el principio, cierto alumnado procedente de minorías culturales puede sentir la escuela como un sitio confuso y extraño, con actividades, prácticas y una lengua que no entienden, desconociendo qué hacer cuando necesitan ayuda e, incluso, qué intención puede haber detrás de lo que dice el profesor o profesora.

Asumir la lógica de los tres puntos expuestos permite argumentar que el fracaso escolar de grupos minoritarios no se puede explicar de forma aislada en términos individuales (como la timidez, pasividad, hiperactividad), cognitivos (retraso intelectual, lingüístico, etc.) o bien sociales (nivel de estudios de los padres, nivel de ingresos, condiciones de vida, etc.), sino en términos sistémicos (Larrauri, 2009; Poveda, 2001). Dicho con otras palabras, las relaciones familia-escuela son 
En la Secretaría de Teoría de la Educación. Revista Interuniversitaria se recibieron durante 2013 un total de 81 trabajos pertenecientes a 109 autores, con procedencia de instituciones científicas extranjeras en el quince por ciento de los casos. De los 81 trabajos recibidos 19 han sido publicados, 58 han sido rechazados y otros 4 están pendientes de publicación. La tasa de rechazo resultante es del 72\%.

El proceso de evaluación de los trabajos ha sido doblemente anónimo, con participación de 50 revisores externos que emitieron un total de 174 informes. El $25 \%$ de los revisores pertenece a instituciones científicas extranjeras.

Araújo, Alberto Filipe

Argos González, Javier

Asensio Aguilera, José María

Azevedo, María da Conceição

Aznar Minguet, Pilar

Bárcena Orbe, Fernando

Bezerra Barbosa, Fátima María

Boavida, João

Buxarrais Estrada, María Rosa

Colom Cañellas, Antoni Joan

Contini, Mariagrazzia

Domínguez Rodríguez, Emilia

Escámez Sánchez, Juan

Etxeberría Balerdi, Félix

Fermoshino, Dores

Fernández March, Amparo

García Aretio, Lorenzo

Gargallo López, Bernardo

Gennari, Mario

Gervilla Castillo, Enrique

Gil Cantero, Fernando

González Faraco, Juan Carlos

Hirsch Adler, Ana Cecilia

Jordán Sierra, José Antonio

Jover Olmeda, Gonzalo

Larrosa Bondía, Jorge

Martínez Martín, Miguel

Masschelein, Jan

Martínez Mut, Bernardo
Universidade do Minho

Universidad de Cantabria

Universidad Autónoma de Barcelona

Universidade de Trás-os-Montes e Alto

Douro

Universidad de Valencia

Universidad Complutense de Madrid

Universidade do Minho

Universidade de Coimbra

Universidad de Barcelona

Universidad de las Islas Baleares

Università di Bologna

Universidad de Extremadura

Universidad de Valencia

Universidad del País Vasco

Universidade de Coimbra

Universidad Politécnica de Valencia

Universidad Nacional de Educación a

Distancia

Universidad de Valencia

Università degli Estudi di Genova

Universidad de Granada

Universidad Complutense de Madrid

Universidad de Huelva

Universidad Nacional Autónoma de

México

Universidad de Barcelona

Universidad Complutense de Madrid

Universidad de Barcelona

Universidad de Barcelona

Katholieke Universiteit Leuven

Universidad Politécnica de Valencia 
ya que orienta a la persona en la línea del comportamiento socialmente normativo y esperado.

Estas discontinuidades entre docentes y padres/madres en relación al sentido, el valor y la cultura escolar pueden repercutir negativamente en el éxito académico de los alumnos, que se encuentran entre dos escenarios simbólicos distintos. Una distancia social y cultural que no se halla entre familias autóctonas de clase media y la cultura y práctica escolar, siendo realidades que están en sintonía y que, mutuamente, retroalimentan su acción, aunque sea tácitamente, al compartir modelos, prácticas, sentidos y valores. Por ejemplo, los docentes esperan de las familias que ayuden a sus hijos en la realización de las tareas académicas. Sin embargo, algunas de estas familias, al no dominar el código o la norma implícita subyacente a la actividad, pueden no saber ayudar a sus hijos o, simplemente, delegar esta tarea a los docentes; pensar que ellos son los responsables ya que confían plenamente en su tarea. No obstante, esta delegación puede percibirse como desinterés por parte de los docentes. Es decir, puede existir una delegación de las tareas escolares por parte de la familia, una delegación que puede explicarse por distintas razones. Para las familias, la confianza en la capacidad de los docentes puede ser absoluta, de manera que les delegan la tarea asociada a los aprendizajes académicos. Para los docentes, sin embargo, esta delegación puede interpretarse, como hemos dicho anteriormente, en un supuesto desinterés que tienen las familias inmigrantes en relación a la escuela (Oller, Esteban-Guitart, Siqués y Vila, 2011). En este caso estaríamos frente a una discontinuidad entre la familia y la escuela basada en la falta de conocimiento entre ambos entornos, lo que conduciría a malentendidos y prejuicios: pensar, por ejemplo, que las familias no tienen ningún interés en la escuela y no creen en la tarea de los profesores, cuando en realidad lo que puede haber es una profunda confianza hacia la tarea que hacen.

Bajo una perspectiva ecológica y sistémica (Bronfenbrenner, 1987; Gifre y Esteban-Guitart, 2012; Larrauri, 2009) es importante enfatizar aquí que los alumnos y alumnas no son, simplemente, sujetos que pertenecen al centro escolar, sino que forman parte de un conjunto de contextos y sistemas: su entorno social, económico, familiar, comunitario, que constituye la "mochila" que traen en el aula. Esta "mochila" puede ser percibida como un límite, un impedimento, un déficit (lingüístico, religioso, económico, étnico), algo negativo y perjudicial (la concepción bajo la cual se sostiene la educación compensatoria). Puede ser concebida, también, como una fuente de conocimientos, habilidades y destrezas susceptibles de ser utilizadas pedagógicamente para vincular la familia (las formas de vida de los alumnos) con la escuela (los "conceptos científicos" que en ella se aprenden).

En un estudio clásico llevado a cabo por Labov (1972), por ejemplo, se demostró que las formas lingüísticas de los niños y niñas de minorías étnicas negras en los Estados Unidos de América eran tan lógicas, complejas y sistemáticas como las formas lingüísticas estándar, sólo que basadas en reglas diferentes que los profesionales de la educación no reconocían. Además, mostró también el impacto 
negativo de las condiciones, contenidos y contextos de las tareas propuestas en las pruebas escolares para los alumnos afroamericanos, habiendo un sesgo que no permitía medir su desempeño real. Precisamente, existe en la actualidad un amplio corpus empírico que muestra las múltiples formas familiares y comunitarias de alfabetización (Gregory, Long y Volk, 2005; Reyes y Esteban-Guitart, en prensa), poniendo en entredicho la existencia de supuestos déficits culturales (González, Moll y Amanti, 2005). Ésta es la perspectiva del proyecto "fondos de conocimiento" que a continuación pasamos a describir con más detalle.

3. El PROGRAMA FONDOS DE CONOCIMIENTO COMO ESTRATEGIA EDUCATIVA PARA CONCILIAR LA PRÁCTICA ESCOLAR CON LA CULTURA FAMILIAR

El origen del programa se remonta al interés en el estudio de las redes de intercambio económico por parte de inmigrantes mexicanos residentes en los Estados Unidos de América. Específicamente, el análisis de las asociaciones rotativas de ahorro y crédito que tienen el objetivo de ayudar económicamente, mediante crédito, a personas mexicanas recién llegadas a los Estados Unidos de América -un sistema conocido como "cundina" o "tanda"-. Mucho más que un fenómeno puramente económico, Vélez-Ibáñez (1983) examinó el pegamento social y cultural que sostiene dichas relaciones transnacionales basadas en lo que llamó confianza mutua (mutual trust) o simplemente confianza. Según el autor, la confianza "da forma a las expectativas de las relaciones dentro de amplias redes de vínculos interpersonales, en las que intimidades, emociones, informaciones, favores, bienes y servicios se intercambian" (Vélez-Ibáñez, 2010, 46). Dicho con otras palabras, la vida transfronteriza depende del establecimiento de relaciones y vínculos sociales, basados en la confianza mutua, que puedan lidiar con la incertidumbre y conflictos generados como resultado de la migración.

Liderado por Vélez-Ibáñez y Greenberg (1992) en la década de los 80 del siglo xx, el "Proyecto Tucson" ("The Tucson Project") tenía el objetivo de estudiar los sistemas de intercambio de la comunidad de origen mexicano de Tucson (Arizona). La metodología llevada a cabo era de corte cualitativo. Más en concreto, los investigadores realizaban entrevistas etnográficas a los hogares de familias de origen mexicano. Posteriormente, se incorporaron Norma González, antropóloga, y Luis Moll, psicólogo educativo, así como distintas maestras como Cathy Amanti. En la década de los 90 se realiza el proyecto fondos de conocimiento propiamente vinculado a la educación ("the Funds of Knowledge for Teaching project"). La idea principal del proyecto era que los hogares y comunidades latinas poseían conocimientos que podían convertirse en poderosos recursos para la práctica escolar (González, Moll, Floyd Tenery, Rivera, Rendón, González y Amanti, 1995; Moll y Greenberg, 1990; Moll, Amanti, Neff y González, 1992).

Un aspecto central era el estudio etnográfico de las familias, una aproximación utilizada para investigar y analizar la historia familiar de los hogares de los alumnos 
de habla hispana, particularmente su historia laboral. La experiencia ha revelado la enorme riqueza de cuerpos de conocimientos acumulados, así como habilidades y estrategias halladas en cada hogar, lo que hemos llamado fondos de conocimiento (González, Andrade y Carson, 2001, 101).

De modo que se entiende por fondos de conocimiento: "Los cuerpos de conocimientos culturalmente desarrollados e históricamente acumulados y destrezas esenciales para el funcionamiento y bienestar familiar o individual" (Moll, 1997, 47). El concepto retiene no solamente las habilidades, destrezas, informaciones y conocimientos que los hogares acumulan resultado de su experiencia vital, sino también las redes sociales de intercambio, retomando la idea original de VélezIbáñez (1983):

Utilizamos la expresión fondos de conocimiento para referirnos a las distintas redes sociales que conectan a las familias con sus entornos sociales y hacen posible compartir o intercambiar recursos, incluidos el conocimiento, las habilidades y el trabajo esencial para su funcionamiento y bienestar (Moll, Tapia y Whitmore, 2001, 186).

Frente a la noción del déficit cultural según la cual los conocimientos y habilidades de las personas dependen de su clase y condición social, el proyecto fondos de conocimiento parte de la premisa según la cual todas las personas -más allá de su condición lingüística, étnica, religiosa, económica- disponen de conocimientos, relaciones, destrezas y habilidades acumuladas en su historia laboral, comunitaria y familiar (González, Moll y Amanti, 2005); así como prácticas de alfabetización (Gregory, Long y Volk, 2005; Reyes y Esteban-Guitart, en prensa). El problema es que en situaciones de diversidad social y cultural, muchas veces estos recursos y actividades son desconocidas $-\mathrm{y}$ consecuentemente silenciadaspor parte de la cultura escolar que reproduce los valores y prácticas del grupo cultural dominante. En este sentido, la idea del proyecto consiste en aproximar la cultura escolar a la cultura familiar a través de la formación de docentes en técnicas antropológicas como la etnografía con el objetivo de visitar los hogares de sus alumnos para documentar los fondos de conocimiento que tienen y poderlos vincular mediante innovaciones curriculares, unidades didácticas o prácticas escolares que permitan contextualizar y extender los conceptos desarrollados en la escuela (González, Moll y Amanti, 2005; McIntyre, Rosebery y González, 2001). Tal y como lo resume recientemente Moll (2011):

En el proyecto fondos de conocimiento, nuestra estrategia consistió en ayudar a los docentes a realizar visitas a las casas de sus alumnos para facilitar el establecimiento de relaciones de confianza con las familias y en la medida de lo posible documentar prácticas sociales, destrezas y conocimientos derivados de las experiencias de estas familias para ser implementadas en la práctica escolar (Moll, 2011, 160). 
El propósito fundamental del trabajo es modificar o mejorar la enseñanza escolar sobre la base del conocimiento y las habilidades que se hallan en las familias locales (Moll, Tapia y Whitmore, 2001, 185).

Ejemplos de "fondos de conocimiento" serían habilidades vinculadas con la agricultura y la cría de animales en familias campesinas, la construcción (carpintería, diseño, arquitectura), la música, la religión, la medicina, la reparación de vehículos o la economía. Conocimientos derivados de la experiencia laboral y la historia familiar de un determinado alumno que se convierten en recursos educativos cuando son incorporados en la actividad escolar por parte de docentes, quienes previamente han documentado estos fondos de conocimiento a través de distintas visitas a las familias. Por ejemplo, Amanti (2005), después de atestiguar que había una gran experiencia en caballos en algunas de las casas de los alumnos y alumnas que visitó, decidió diseñar una unidad curricular en torno a ello utilizando distintos recursos para trabajar distintas materias. En ciencias sociales, por ejemplo, se trató el tema de las misiones de los españoles y su rol en traer caballos a América, se visualizó una película sobre vaqueros, y se estudiaron los distintos usos de los caballos. En el área de lenguaje y arte se creó un diccionario español-inglés sobre distintos conceptos vinculados al tema, se analizó un vídeo, se buscaron libros, cuentos o historias sobre caballos. En el área de ciencia y matemáticas se analizó la anatomía del caballo, su conducta, su evolución, así como se trabajó con distintas unidades de medida y transformación. Otro ejemplo es el trabajo de Sandoval-Taylor (2005) en el que se diseñó un modulo de enseñanza y aprendizaje basado en la construcción. La maestra descubrió que en muchas de las familias de sus alumnos y alumnas (donde un 50\% era minoría hispánica, un 40\% indígenas norteamericanos y un $10 \%$ norteamericanos) había gran cantidad de conocimientos vinculados a la construcción. El tema era "¿cómo construir una casa?". A partir de esta pregunta, se realizaron varias actividades como, por ejemplo, leer el cuento de los tres cerditos, hacer que los estudiantes dibujasen su casa, realizar un diccionario vinculado a la construcción, construir una casa proporcional para una muñeca, analizar distintas fotografías de casas o construir casas con distintas figuras geométricas.

A pesar de los efectos positivos del programa, descritos brevemente en la introducción, recientemente se han señalado algunas limitaciones o aspectos a mejorar (Moll, 2005; Ríos-Aguilar, Kiyama, Gravitt y Moll, 2011). Una de las críticas más recurrentes es la dificultad de llevar a cabo un análisis exhaustivo de los fondos de conocimiento de todos los alumnos. Por lo general, se eligen dos o tres alumnos y se generalizan sus fondos de conocimiento. Uno puede pensar que ello no representa a todos los alumnos, sin embargo, se escogen aquellos que pueden ser más representativos, como se ha demostrado en los ejemplos anteriormente mencionados de Amanti y Sandoval-Taylor. También es verdad que la práctica escolar corriente tampoco representa a todas las culturas presentes en la geografía escolar, de modo que muchas veces la inclusión de ellas puede estimular ejercicios de contraste y ejemplificación. Por ejemplo, pueden detectarse fondos de conocimiento vinculados a la historia política de Rumanía y, a través de ellos, tratar 
LA PRÁCTICA EDUCATIVA DESDE LA PERSPECTIVA DE LOS FONDOS DE CONOCIMIENTO E IDENTIDAD

la historia política de España así como distintas formas de gobierno. En definitiva, se trata de incorporar las "mochilas" culturales de los alumnos como mecanismo para poder extender, incrustar y ejemplificar las tareas y contenidos escolares.

A continuación destacamos, muy brevemente, algunas de las limitaciones que los propios protagonistas del desarrollo del programa educativo han detectado. Lo que nos permitirá introducir una expansión de la intervención a través de la noción de fondos de identidad.

\subsection{Algunas limitaciones del programa educativo fondos de conocimiento}

Recientemente, Ríos-Aguilar, Kiyama, Gravitt y Moll (2011) han señalado tres limitaciones o aspectos a considerar para mejorar la propuesta inicial. En primer lugar, en la literatura al respecto existe un énfasis excesivo en los fondos de conocimiento fuera de parámetros culturales como la consideración de la clase social. En segundo lugar, para detectar los fondos de conocimiento se ha utilizado básicamente una única aproximación metodológica (la etnografía a través de las visitas y entrevistas que realizan los docentes a los hogares de sus alumnos). Finalmente, existe una reducción terminológica y epistémica al focalizar los fondos de conocimiento en los saberes y prácticas familiares. Es decir, la principal unidad de análisis es la familia.

En relación al primer punto, los estudios sobre fondos de conocimiento generalmente no reconocen el impacto de las relaciones de poder en las instituciones educativas. En este sentido, Moll (2005) lamenta que en la investigación sobre los fondos de conocimiento, con los docentes como colaboradores, no se hayan desarrollado análisis más sofisticados sobre la clase social, en realidad un factor que no suele tenerse en cuenta. Según Moll (2005), los recursos incrustados en los hogares y las prácticas de la escuela están siempre estrechamente relacionados con cuestiones más amplias que tienen que ver con la clase social, la ideología y el poder. Aspectos que siguiendo la teoría ecológica de Bronfenbrenner (1987) se conocen como factores macroculturales (Ratner, 2011), es decir, instituciones sociales, creencias y artefactos culturales que configuran y diseñan los patrones de conducta psicológica y actividad social. Por ejemplo, se espera al entrar en una iglesia asumir unos determinados patrones de conducta.

Otra limitación detectada se encuentra relacionada con la aproximación metodológica utilizada. A pesar de que la metodología cualitativa, en general, y la etnografía, en particular, parece ser el modo más adecuado de detectar los fondos de conocimiento de las familias, Ríos-Aguilar (2010) ha sugerido la inclusión de procedimientos cuantitativos que permiten, además de detectar los fondos de conocimiento, relacionarlos estadísticamente con distintas conductas como el rendimiento académico, la alfabetización, la participación ciudadana o el éxito profesional.

Finalmente, se ha criticado el hecho de reducir el análisis de los fondos de conocimiento a las prácticas, rutinas y valores familiares. Como reconoce el mismo 
LA PRÁCTICA EDUCATIVA DESDE LA PERSPECTIVA DE LOS FONDOS DE CONOCIMIENTO E IDENTIDAD

Moll (2005), hasta el momento la investigación se ha centrado, casi exclusivamente, en las prácticas adultas y sus relaciones. Sin embargo, los niños y niñas también crean sus mundos sociales y sus fondos de conocimiento, que además pueden ser independientes de los propios de la vida adulta.

Precisamente con el objetivo de superar estas limitaciones se ha propuesto la noción de fondos de identidad, concepto que pasamos brevemente a describir e ilustrar.

\section{LA PROPUESTA DE LOS FONDOS DE IDENTIDAD}

En el proceso del desarrollo cultural, el niño va asimilando no sólo el contenido de la experiencia cultural, sino también los métodos y modos de la conducta cultural y del pensamiento: va dominando los particulares medios culturales creados por la humanidad en el curso del desarrollo histórico, por ejemplo, el lenguaje, los símbolos aritméticos, etc. El niño aprende a emplear funcionalmente determinados signos como medio para ejecutar tal o cual operación psicológica. De tal manera, las formas elementales y primitivas de conducta se convierten en actos y procesos culturales mediados (Vygotksi 1997, 347).

El concepto de fondos de identidad no nace con la voluntad de sustituir el de fondos de conocimiento, más bien de complementarlo a la luz de las críticas brevemente expuestas en el apartado anterior. Dicho con otras palabras, las tres limitaciones anteriormente mencionadas han motivado la extensión del concepto inicial de fondos de conocimiento.

Por un lado, dicho concepto debe ampliarse para incluir los factores macroculturales: instituciones sociales, artefactos y creencias o conceptos culturales (ideología), considerados el origen y la raíz de la conducta humana culturalmente mediada, organizada y regulada (Ratner, 2011). Moll (2005) y Ríos-Aguilar et al. (2011) se refieren a ello cuando recomiendan considerar la clase social, la ideología y las relaciones de poder como aspectos incrustados y subyacentes a las prácticas escolares, así como las realizadas en el hogar. En este sentido, RíosAguilar y colaboradores (2011) proponen combinar la aproximación de los fondos de conocimiento con las teorías sobre el capital social y cultural. Ello permite considerar, por ejemplo, cómo ciertas dinámicas de poder en las instituciones educativas permiten el aprovechamiento y valoración de las formas de vida de los alumnos. El objetivo final es obtener una mejor comprensión del rendimiento académico de los llamados "estudiantes subrepresentados" o "infrarrepresentados" ("under-represented students") (Ríos-Aguilar et al., 2011), es decir, aquellos que poseen alguna de las siguientes características: bajos ingresos, son de un grupo étnico minoritario, de origen extranjero (inmigrantes, indocumentados, inmigrantes de segunda generación) y/o tienen un acento extranjero.

Por otro lado, la extensión del concepto fondos de conocimiento debe incluir la utilización de distintas metodologías con el objetivo de superar el 
"carácter uni-metodológico" que ha tenido la investigación hasta el momento, salvo en contadas excepciones (Ríos-Aguilar, 2010).

Finalmente, el concepto en cuestión debe extender su objeto de análisis. Es decir, los estudios sobre fondos de conocimiento centran su análisis en el estudio de las prácticas, creencias y valores familiares a través de las visitas que se realizan a los hogares de los alumnos. Ahí, se realizan distintas entrevistas con el objetivo de detectar fondos de conocimiento. En este sentido, se pregunta, por ejemplo, sobre la historia de la migración de la familia -si es pertinente-, su historia laboral o las lenguas que hablan y bajo qué circunstancias.

Teniendo en mente estas tres exigencias se sugiere el concepto de fondos de identidad (Esteban-Guitart, en prensa). Se entiende por fondos de identidad aquellos artefactos, tecnologías o recursos, históricamente acumulados, culturalmente desarrollados y socialmente distribuidos y transmitidos, esenciales para la autodefinición, autoexpresión y autocomprensión de las personas. Dicho con otras palabras, los fondos de identidad son los fondos de conocimiento que las personas utilizan para autocomprenderse y autodefinirse. Por ejemplo, un fondo de conocimiento de una familia puede ser el fútbol ya que el padre es un amante de dicho deporte y además ejerce de árbitro. Sin embargo, puede no ser un fondo de identidad de un alumno si dicha afición no está incorporada en su identidad -la definición que hace sobre sí mismo y aquello que para él es significativo-. Cuando un alumno incluye en su autodefinición el fútbol, incluso participa de dicha actividad al practicarlo, por ejemplo, entonces el fondo de conocimiento familiar se convierte en fuente de identidad; en recurso a través del cual la persona se define, expresa y realiza. En este caso, podría realizarse una actividad académica vinculada a las matemáticas a partir de la transformación de un campo de fútbol real a un campo de fútbol para playmobils $@$. Al estar incorporado en las formas de vida y de sentido del alumno, la actividad sería significativa y, consecuentemente, el aprendizaje sería más profundo.

Al igual que el fútbol pueden aparecer otros recursos como, por ejemplo, la religión, la música o cualquier dispositivo que forme parte del sentido del aprendiz, es decir, aquello que a uno le importa y le es significativo. En particular, pueden distinguirse cinco grandes tipos de fondos de identidad, a saber: 1) Fondos geográficos de identidad (cuando se utiliza un determinado espacio físico como un pueblo, ciudad, montaña, río para autodefinirse o definir un colectivo como, por ejemplo, el Cañón del Sumidero en el caso de la identidad chiapaneca); 2) Fondos sociales de identidad (incluye las redes sociales del alumno, aquellas personas que considera significativas y que se convierten en recursos para su desarrollo); 3) Fondos culturales de identidad (la utilización de artefactos culturales como una bandera o un himno nacional para autoexpresarse o autodefinirse); 4) Prácticas de identidad (actividades que uno realiza y que suelen ser significativas como practicar un deporte, escuchar música o conectarse a Internet). Finalmente, 5) Fondos institucionales de identidad (la Iglesia católica, el matrimonio, la familia o el trabajo pueden convertirse en dispositivos que la persona utiliza para entender 
el mundo y regular su conducta en él, aunque pueda no ser consciente de ello, es decir, uno puede no ser consciente de la influencia de una determinada ideología en su conducta y experiencia psicológica). A través de la participación en distintas situaciones sociales (prácticas culturalmente guiadas) los niños y niñas van incorporando -se van apropiando- de distintos conocimientos que se convierten en testimonios, materias, documentos o memorias distribuidas de identidad.

Es necesario destacar tres cosas de la definición propuesta. En primer lugar, la mediación "múltiple-cultural" de la identidad humana. Es decir, personas, símbolos, paisajes geográficos, actividades o instituciones forman parte de la identidad humana, siempre distribuida y situada social y culturalmente. Según Vygotski (1979) nuestra relación con el medio está culturalmente mediada. Es decir, las personas superan sus restricciones biológicas y autorregulan su conducta a través de la modificación cultural del entorno. Por ejemplo, ponemos el despertador para levantarnos, cruzamos un paso de peatones cuando el semáforo está en verde, rezamos con la ayuda de una cruz o animamos a nuestro equipo de fútbol con una bandera. Todos estos artefactos culturales, a la vez físicos y simbólicos, permiten el desarrollo de lo que el autor llamó "funciones psicológicas superiores". Por ejemplo, con la ayuda de un bloc de notas organizamos nuestro pensamiento, a través de un "power point" amplificamos nuestra memoria o gracias a un telescopio vemos las estrellas. Estas "prótesis culturales" (Bruner, 1990) amplifican nuestros recursos y capacidades psicológicas: el pensamiento, la memoria, la percepción. Según Scribner (1990, 92) el gran mérito de Vygotski consiste, precisamente, en dar un contenido y valor psicológico a lo que nos rodea, más allá de las fronteras del organismo:

El don especial de Vygotski fue el de poder captar el significado de lo social tanto en las cosas como en las personas. El mundo en que vivimos está humanizado, repleto de objetos materiales y simbólicos (signos, sistemas de conocimientos) que están construidos culturalmente, de origen histórico, y de contenido social. Ya que todas las acciones humanas, incluyendo los actos de pensar, implican la mediación de tales objetos (herramientas y signos) son, solo por esto, de una esencia social.

Siguiendo esta perspectiva, debemos entender la identidad humana, la definición que hacemos de nosotros mismos, como un producto culturalmente mediado. Es decir, una cosa que no ocurre exclusivamente "dentro de" nuestras cabezas sino sobre todo "fuera" de ellas -ocurre a partir de ellas-. En este sentido, se ha propuesto el concepto de "cognición distribuida" (Salomon, 2001) para integrar, en una misma unidad de análisis, la persona y el mundo. Mutatis mutandis, podríamos considerar que la identidad está distribuida entre la subjetividad de una persona pero también todo aquello que utiliza: otras personas significativas, artefactos culturales (instrumentos físicos, creencias religiosas, prácticas reguladas), instituciones sociales. Es en este sentido que debe entenderse el concepto de fondos de identidad. 
En segundo lugar, e implícito en la consideración anterior, el carácter histórico de la identidad. Todos los "fondos de identidad" anteriormente descritos son productos mediados históricamente. El catolicismo, por ejemplo, como artefacto ideológico e institución social es un producto histórico; al igual que una determinada profesión, una determinada montaña, ciudad o valle, o una persona (la pareja, por ejemplo), que cambia con la edad.

Finalmente, se hace necesario destacar el tema de la "transmisión". Si la identidad humana supone la apropiación y utilización de fondos distribuidos de identidad, la educación -socialización- es el proceso a través del cual se da esta apropiación. Es decir, es a través de las otras personas (Moll 2001) como aprendemos a utilizar, e incorporamos, estos artefactos, prácticas y significados asociados a una determinada institución social. Por eso, los fondos de identidad están socialmente distribuidos y transmitidos. El andamiaje social -la educación, ya sea formal o informal- es lo que convierte unos determinados fondos de conocimiento en fondos de identidad. La diferencia entre ambos conceptos es que mientras que los primeros forman parte de un determinado contexto de actividad, una familia, los segundos están, necesariamente, interiorizados por una persona que alude a ellos a la hora de autoentenderse, autodefinirse y autoexpresarse. Evidentemente la educación no supone nunca un viaje en solitario, ni una simple y pasiva asimilación del medio; más bien conlleva la participación activa en contextos de actividad, la conexión sustancial entre informaciones nuevas con conocimientos ya adquiridos, así como la utilización de artefactos que pueden ser "físicos", como un ordenador, o simbólicos, como una ecuación matemática. Aclarando que el ordenador es también un producto simbólico, y la ecuación matemática tiene una repercusión física.

\section{1. ¿Cómo detectar los fondos de identidad?}

Con el objetivo de detectar los fondos de identidad de los alumnos pueden utilizarse distintas estrategias y metodologías. Más concretamente, a partir de la "multimetodología autobiográfica" de Bagnoli (2004) se ha desarrollado una estrategia cualitativa más amplia -la "multimetodología autobiográfica extendida" (Esteban-Guitart, 2012) - que ofrece quince técnicas para el estudio de la identidad organizadas alrededor de cuatro dimensiones, a saber: entrevistas en profundidad, el dibujo identitario revisado, la detección de artefactos-rutinas-formas de vida y la creación de mapas psicogeográficos (Esteban-Guitart, 2012). Dos de estas técnicas son: el "dibujo identitario" y el "círculo significativo".

En el primer caso, consiste en responder, mediante un dibujo, a la siguiente instrucción: "Podrías intentar dibujar quién piensas que eres en este momento de tu vida. Puedes añadir, si quieres, las personas y cosas que para ti sean significativas en este momento de tu vida" (Bagnoli, 2004; Gifre, Monreal y Esteban-Guitart, 2011). A continuación se pide al entrevistado que explique lo que ha dibujado. El 
LA PRÁCTICA EDUCATIVA DESDE LA PERSPECTIVA DE LOS FONDOS DE CONOCIMIENTO E IDENTIDAD

objetivo no es interpretar su dibujo, sino simplemente poder describir los recursos que la persona utiliza para autodefinirse.

Figura 1. EJEMPlo De Dibujo identitario de unA NiÑa DE 10 AÑoS

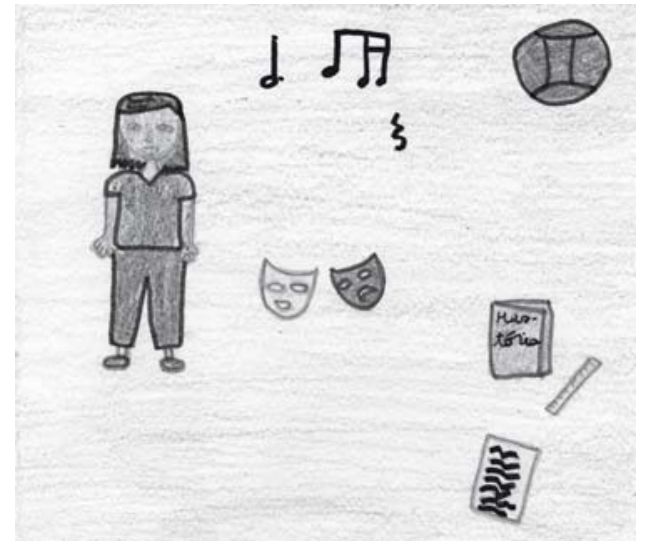

En el ejemplo que aparece en la Figura 1, se puede observar cómo una niña de 10 años se ha dibujado con aquellas cosas que le gustan. Según su explicación:

Me veo una persona alegre, lista. Me gusta la música, el teatro, la historia, contar leyendas o cuentos. He hecho natación, vela, escalada, kayak. No todos los deportes me gustan, por ejemplo no juego a futbol pero cuando veo jugar el Barça estoy nerviosa por saber si ganará o perderá. En el colegio me lo paso muy bien, a veces me gustaría decir secretos que no puedo decir, otras veces me da vergüenza explicarlos.

En este caso, se pueden ver, básicamente, distintas prácticas de identidad al aparecer distintas actividades como la música, el deporte, o la historia. En este sentido, destaca en el dibujo la presencia de fondos culturales de identidad como la pelota o el libro de historia.

Lo que esta técnica nos permite detectar, de una manera sencilla y rápida, son los intereses, experiencias y conocimientos integrados en la definición de una persona, en este caso una niña de 10 años de edad. Podemos suponer que la música, el teatro, la historia, las narraciones y los deportes pueden ser motivos a partir de los cuales diseñar actividades que serán significativas para el alumno. Por supuesto, el dibujo es una excusa para indagar los conocimientos, saberes y destrezas acumulados de la persona entrevistada. A partir de aquí pueden, siguiendo la filosofía del programa fondos de conocimiento, diseñar actividades educativas a partir de los fondos de identidad hallados.

Por ejemplo, Saubich y Esteban-Guitart (2011) documentaron distintos fondos de identidad de una alumna de origen marroquí de sexto grado de primaria 
escolarizada en el sistema público educativo español a partir de los cuales diseñaron e implementaron distintas actividades como conocimiento geográfico a través de la comparación entre Marruecos y España, un taller de cocina con ingredientes propios de la cocina tradicional marroquí o actividades alrededor de la henna, una planta utilizada para adornar las manos y los pies de las novias en países como India, Marruecos o Pakistán. Algunos de los fondos de identidad detectados fueron: conocimientos geográficos de África y España derivados de la situación transnacional de la alumna (fondos geográficos de identidad), destrezas en la música, la cocina y el baile (prácticas de identidad), la presencia de artefactos como la comida tradicional marroquí, la henna o Internet (fondos culturales de identidad), la importancia de la familia (fondos sociales de identidad) y la influencia del Islam (fondos institucionales de identidad).

Otra técnica o estrategia de investigación cualitativa complementaria, para acceder a los fondos de identidad de un alumno o alumna, es el "círculo significativo". En este caso, la tarea consiste en dibujar, en medio de un círculo, las personas significativas (representadas por redondeles) y las actividades, aficiones, instituciones relevantes para el entrevistado (en cuadrados), teniendo en cuenta que cuanto más al centro, más importantes son para la persona. Esta técnica puede servir, también, para detectar los fondos de conocimiento de los padres y, por supuesto, los fondos de identidad de los hijos. En el ejemplo que aparece en la Figura 2, una madre dibuja lo que define como "su círculo emocional", en el que incluye, en el centro, a su pareja y padres, así como la danza. También, más alejado del centro, representa a sus amigos y la familia extensa. La danza, por ejemplo, es un fondo de conocimiento que puede ser, o no, un fondo de identidad si su hija/o, el alumno/a del docente, lo representa en su dibujo identitario o círculo significativo, es decir, si comparte esta afición o actividad y lo utiliza como medio a través del cual definirse y expresar aquello que para él o ella es importante, significativo.

Figura 2. EJEMPlo de CírCulo Significativo de una Madre De 41 años

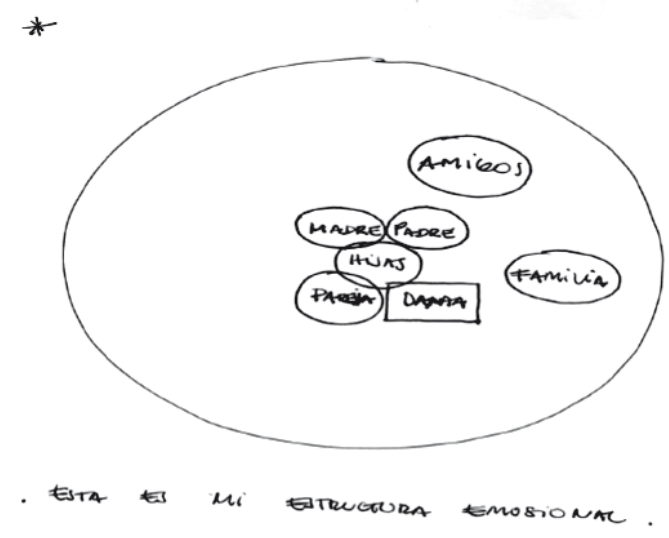


Figura 3. EJEMPlos de CíRCulos SignificATIVOS DE DOS HERMANOS, UNA CHICA DE 5 AÑOS (IZQUIERDA) Y OTRA DE 8 (DERECHA)
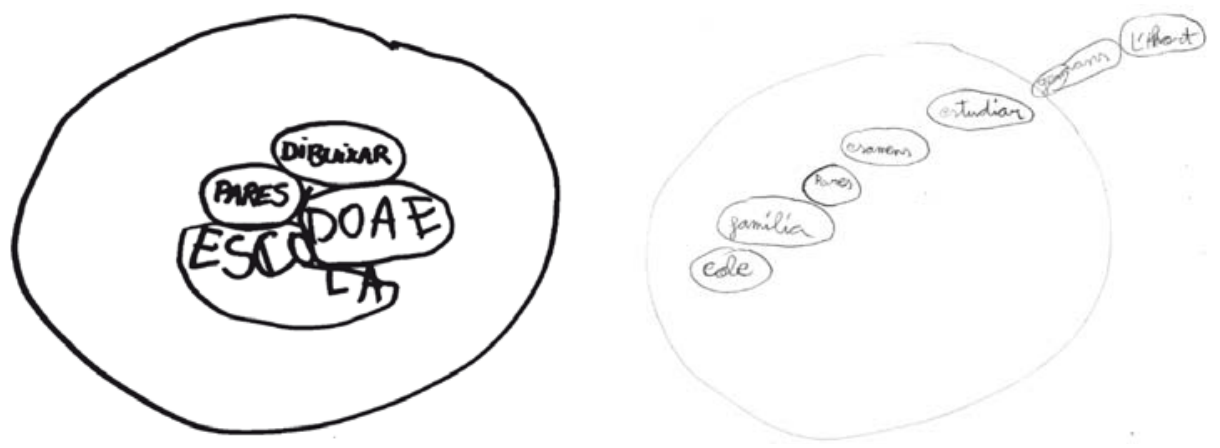

En la Figura 3 aparecen dos ejemplos de círculos significativos, en este caso corresponden a dos hermanas, una de 5 años y la otra de 8. En ambos casos destacan elementos comunes: la escuela y la familia (los padres). Se trataría de un fondo institucional de identidad, en el primer caso, y un fondo social de identidad, en el segundo. También aparecen elementos diferenciadores como el dibujo ("dibuixar") en el caso de la niña de 5 años y el huerto ("'hort") en la hermana mayor.

En definitiva, técnicas como las expuestas permiten, de un modo fácil y rápido, detectar las fuentes de sentido e identidad del estudiante. Dichos recursos identitarios son de naturaleza histórica y cultural. En realidad, son dispositivos culturales que moldean la conducta humana al convertirse en modelos de identidad. El objetivo no es otro que documentarlos con el objetivo de diseñar, posteriormente, actividades educativas propicias para el aprendizaje de distintos contenidos escolares. Se espera que al reconocer los intereses, destrezas y saberes del alumnado se propicie su implicación, motivación, así como el establecimiento de puentes con los contenidos escolares.

\section{DISCUSIÓN Y CONCLUSIONES FINALES}

Etxeberría y Elosegui (2010) destacan distintos factores que pueden subyacer al fracaso escolar del alumnado procedente de familias inmigrantes. A los problemas en la adquisición de la(s) lengua(s) de la escuela debido a una casi nula atención a las culturas de origen del alumnado inmigrante, se añade la concentración del alumnado inmigrante en los centros públicos, un endurecimiento de la legislación europea y española con respecto a los inmigrantes y sus condiciones de vida, así como la existencia de un rechazo a los inmigrantes (una gran parte de alumnos, profesores y familias se muestran contrarios y contrarias a la presencia de inmigrantes en las aulas). Finalmente, los autores destacan otro factor vinculado 
a la limitada formación de los profesores en relación a la atención educativa a la diversidad, así como sus expectativas negativas en relación al futuro y su práctica profesional. En la revisión de García, Gómez y Bouachra (2008, 38) aparece, además, la relación familia-escuela como «uno de los factores más importantes en la integración escolar de este alumnado".

En este sentido, el programa fondos de conocimiento -y su extensión a través de la noción de fondos de identidad- puede contribuir a mejorar tres de los factores anunciados anteriormente, a saber: 1) el rechazo de actitudes racistas por parte de la cultura escolar hacia las familias inmigrantes, así como por parte de ellas en relación a los docentes y la escuela, 2) la formación docente alrededor de una estrategia educativa que se ha mostrado eficaz para gestionar la diversidad social y cultural del alumnado y sus familias y 3) la mejora de las relaciones familiaescuela. Pensamos que la incorporación de los fondos de identidad de los alumnos en espacios de educación formal puede ser una vía que permita convertir los fondos de conocimiento en recursos pedagógicos para optimizar los aprendizajes. A diferencia de otros enfoques y estrategias de atención a la diversidad, por ejemplo la educación compensatoria, se supone una riqueza intrínseca a las personas, más allá de su condición lingüística, económica, religiosa. El problema radica en que estas formas culturales de conocimiento pueden ser invisibles para la cultura escolar, lo que conlleva una discontinuidad entre ésta y las formas informales de socialización de algunos grupos culturalmente minoritarios. Por lo tanto, el fracaso escolar en determinados colectivos culturales puede explicarse por la infravaloración, incluso rechazo o desconocimiento, que tiene la cultura escolar en relación a las múltiples formas de vida y fondos de conocimiento de sus alumnos. Dicho con otras palabras, no es que las personas que proceden de grupos minoritarios tengan ambientes culturalmente deprimidos, sino que sus contextos de conocimiento, vida y actividad no están presentes en la escuela.

Se trata de una estrategia pedagógica congruente con el "paradigma de las continuidades-discontinuidades familia-escuela" (Poveda, 2001) que contribuye a la literatura alrededor de la educación inclusiva próxima a la instrucción culturalmente sensible" (culturally responsive instruction), "congruencia cultural en la instrucción" (cultural congruence in instruction) o la llamada "enseñanza basada en la cultura" (culture-based teaching) (Esteban-Guitart, Oller, Vila y Quintanilla, 2012; McIntyre, Kyle y Rightmyer, 2005). El supuesto es que si se aproxima la organización de la actividad escolar a la experiencia y formas de vida de los alumnos y alumnas, se reconoce su legado cultural (sus fondos de conocimiento e identidad), se facilita la transición escuela, familia y entorno social, mejorando la implicación, motivación y sentido del contexto educativo escolar y lo que en él se hace. Por supuesto, futuras investigaciones son necesarias para evaluar el impacto que tiene la práctica educativa basada en los fondos de identidad, además de perfilar cómo puede llevarse a cabo en materias curriculares específicas como matemáticas, biología o lengua y literatura. 
Sin embargo, como toda práctica educativa, la educación desde los fondos de conocimiento e identidad supone algunas limitaciones o dificultades en su implementación. En primer lugar, conocer empíricamente a los alumnos y sus familias requiere trabajo, tiempo y esfuerzo. Además, existen en las aulas españolas una gran heterogeneidad de alumnos que proceden de distintos entornos sociales y culturales, ello dificulta la generalización de los fondos de conocimiento e identidad hallados en algunos de ellos. Otra dificultad estriba en las concepciones esencialistas que reducen el término cultura a aspectos excesivamente folclóricos, inmutables y permanentes. Contrariamente, se entiende por cultura las experiencias y prácticas de los alumnos, es decir, "lo que la gente hace y dice sobre lo que hace" - "What it is that people do, and what they say about what they do"- (González, 2005, 40). Quizá el escollo principal sea el cambio de paradigma o cultura que supone en la formación y práctica docente. Es decir, el hecho, por ejemplo, de trascender las relaciones familia-escuela tradicionales basadas en las tutorías unidireccionales en las que el docente, tutor, informa a las familias del desarrollo y desempeño académico de sus hijos. A pesar de estas limitaciones o dificultades, pensamos que merece la pena considerar dicha aproximación como una estrategia, de entre otras, para gestionar eficazmente la diversidad existente en la actualidad en la población y geografía escolar.

A modo de conclusión y síntesis final, creemos que se trata de una estrategia que intenta garantizar el acceso, permanencia y promoción en el sistema educativo del alumnado en situación de desventaja social a través de la modificación de las relaciones de poder escuela-familia y el reconocimiento del alumnado y sus contextos informales de vida. Ello se enmarca en una concepción educativa que considera beneficioso que la escuela se inserte en una red de relaciones educativas más amplia que permita articular distintos contextos de enseñanza y aprendizaje para poder apoyar, contextualizar y extender los aprendizajes escolares (Vila, 1998; Vila y Casares, 2009).

\section{REFERENCIAS BIBLIOGRÁFICAS}

Amanti, C. (2005) Beyond a beads and feathers approach, en González, N.; Moll, L. y Amanti, C. (eds.) Funds of knowledge. Theorizing practices in housebolds, communities, and classrooms. Mahwah, Lawrence Erlbaum Associates, 131-141.

ANDREWS, J. y YEE, C. W. (2006) Children's "funds of knowledge" and their real life activities: Two minority ethnic children learning in out-of-school contexts in the UK. Educational Review, 58 (4), 435-49.

Bagnoli, A. (2004) Researching identities with multi-method autobiographies. Sociological Research Online, 9 (2), 1-15.

BROKER, L. (2003) Learning how to learn. Parental ethnotheories and young children's preparation for school. International Journal of Early Years Education, 11 (2), 117-128.

Bronfenbrenner, U. (1987) La ecología del desarrollo bumano. Experimentos en entornos naturales y diseñados. Barcelona, Paidós.

Bruner, J. (1990) Acts of meaning. Cambridge, Harvard University Press. 
LA PRÁCTICA EDUCATIVA DESDE LA PERSPECTIVA DE LOS FONDOS DE CONOCIMIENTO E IDENTIDAD

ERICKSON, F. (1982) Classroom discourse as improvisation: Relationship between academia task structure and social participation structure in lessons, en WiLKINSON, L. S. (coord.) Communicating in the classroom. New York, Academia Press, 153-181.

EstebAn-Guitart, M. (2012) La multi-metodología autobiográfica extendida. Una estrategia cualitativa para estudiar la identidad, los fondos de conocimiento y las formas de vida. Revista Electrónica de Metodología Aplicada, 17 (2), 51-64.

- (en prensa) Funds of identity, en TeO, T. (ed.), The Encyclopedia of Critical Psychology. New York, Springer.

Esteban-Guitart, M.; Oller, J.; Vila, I. y Quintanilla, L. (2012) Culture-based teaching. An example of using families' funds of knowledge to mediate early years learning. Póster presentado en la Conferencia Anual de la European Early Childhood Education Research Association, celebrada en Oporto (Portugal) del 29 de agosto al 1 de septiembre.

EtXeberría, F. y Elosegui, K. (2010) Integración del alumnado inmigrante: obstáculos y propuestas. Revista Española de Educación Comparada, 16, 235-263.

GarCía, F. J.; Gómez, M. R. y BouAchra, O. (2008) Población inmigrante y escuela en España: un balance de investigación. Revista de Educación, 345, 23-60.

Gifre, M. y Esteban-GuitarT, M. (2012) Consideraciones educativas de la perspectiva ecológica de Urie Bronfenbrenner. Contextos Educativos, 15, 79-92.

Gifre, M.; Monreal, P. y Esteban-Guitart, M. (2011) El desarrollo de la identidad a lo largo del ciclo vital. Un estudio cualitativo y transversal. Estudios de Psicología, 32 (2), 227-241.

GONZÁLEZ, N. (2005) Beyond culture: The hibridity of funds of knowledge, en GONZÁLEZ, N.; Moll, L. y AmantI, C. (eds.) Funds of knowledge. Theorizing practices in households, communities, and classrooms. Mahwah, NJ, Lawrence Erlbaum Associates, 29-46.

GonZÁlez, N.; ANDRAdE, R. y CARSON, C. (2001) Creating links between home and school mathematics practices, en MCInTyre, E.; Rosebery, A. y GonZÁlez, N. (eds.) Classroom diversity. Connecting curriculum to students' lives. Porstmouth, NH, Heinemann, 100-114.

GonZÁleZ, N. y Moll, L. (2002) Cruzando el puente: Building Bridges to funds of knowledge. Educational Policy, 16 (4), 623-641.

GonzÁlez, N.; Moll, L. y Amanti, K. (2005) Funds of knowledge: Theorizing practices in bouseholds, communities, and classrooms. Mahwah, NJ, Lawrence Erlbaum Associates.

González, N.; Moll, L.; Floyd Tenery, M.; Rivera, A.; Rendón, P.; GonzÁlez, R. y Amanti, C. (1995) Funds of knowledge for teaching in latino households. Urban Education, 29 (4), 443-471.

Gregory, E.; LONG, S. y VolK, D. (2005) Many pathways to literacy. Young children learning with siblings, grandparents, peers and communities. New York, Routledge.

Hinojal, L.; MAsó, S.; Turon, E. y Esteban-GutTart, M. (2012) Identidad, fondos de conocimiento y formas de vida de la familia Cardona Rodríguez. Un estudio psicogeográfico. Papeles de Trabajo sobre Cultura, Educación y Desarrollo Humano, 7 (3), 1-28.

HoGG, L. (2011) Funds of knowledge: An investigation of coherence within the literature. Teaching and Teacher Education, 27 (3), 666-677.

LARRAuri, R. C. (2009) Ecosistema educativo y fracaso escolar. Revista Iberoamericana de Educación, 49 (4), 2-9.

LabOv, W. (1972) Language in the inner city. Philadelphia, University of Pennsylvania Press.

Marshall, E. y ToOhey, K. (2010) Representing family: Community, funds of knowledge, bilingualism and multimodality. Harvard Education Review, 80 (2), 221-41. 
LA PRÁCTICA EDUCATIVA DESDE LA PERSPECTIVA DE LOS FONDOS DE CONOCIMIENTO E IDENTIDAD

Mcintyre, E.; Kyle, D. y Rightmyer, E. (2005) Los fondos de conocimiento de las familias como mediación de la enseñanza en los colegios rurales. Cultura y Educación, 17 (2), $175-195$.

McIntyre, E.; Rosebery, A. y GonZÁlez, N. (2001) Classroom diversity. Connecting curriculum to students' lives. Porstmouth, $\mathrm{NH}$, Heinemann.

McIntyre, E.; Rosebery, A. y GonzÁlez, N. (2001) Connecting students' cultures to instruction, en McIntyre, E.; Rosebery, A. y GonzÁlez, N. (eds.) Classroom diversity. Connecting curriculum to students' lives. Porstmouth, NH, Heinemann, 1-13.

MolL, L. C. (ed.) (1990) Vygotsky and education: Instructional implications and applications of socio-historical psychology. Cambridge, uK, Cambridge University Press.

- (1997) Vygotsky, la educación y la cultura en acción, en Álvarez, A. (ed.) Hacia un currículum cultural. La vigencia de Vygotski en la educación. Madrid, Fundación Infancia y Aprendizaje, 39-53.

- (2001) Through the mediation of others: Vygotskian research on teaching, en RICHARDson, V. (ed.) Handbook of Research on Teaching. Washington, American Educational Research Association, 111-129.

- (2005) Concluding commentary. Reflections and possibilities, en González, N.; Moll, L. y Amanti, C. (eds.) Funds of knowledge. Theorizing practices in households, communities, and classrooms. Mahwah, NJ, Lawrence Erlbaum Associates, 275-287.

- (2011) Only Life Educates: Immigrant Families, the Cultivation of Biliteracy, and the Mobility of Knowledge, en PORTES, P. y SALAS, S. (eds.) Vygotsky in 21st-Century Society: Advances in Cultural Historical Theory and Praxis with Non-Dominant Communities. New York, Peter Lang, 151-161.

- (2013) L. S. Vygotsky and education. New York, NY, Routledge.

Moll, L. y GreEnBerg, J. (1990) Creating zones of possibilities: Combining social contexts for instruction, en Moll, L. (ed.) Vygotsky and education. Cambridge, uK, Cambridge University Press, 319-348.

Moll, L.; TAPia, J. y Whitmore, F. (2001) Conocimiento vivo: la distribución social de los recursos culturales para el pensamiento, en SALOMON, G. (comp.) Cogniciones distribuidas. Consideraciones psicológicas y educativas. Buenos Aires, Amorrortu, 185-213.

Moll, L.; Amanti, C., NefF, D. y GonZález, N. (1992) Funds of knowledge for teaching: using a qualitative approach to connect homes and classrooms. Theory into Practice, 31 (2), 132-141.

OGBu, J. (1982) Cultural discontinuities and schooling. Anthropology and Education Quarterly, 13 (4), 290-307.

OGBu, J. y Simons, H. (1998) Voluntary and involuntary minorities: A cultural-ecological theory of school performance with some implications for education. Anthropology $\&$ Education Quarterly, 29 (2), 155-188.

Oller, J.; Esteban-Guitart, M.; Siqués, C. y Vila, I. (2011) Exploring home and school continuities and discontinuities. Ethnotheories of teachers and African parents in Catalonia, Spain. Comunicación presentada al III Congreso ISCAR, celebrado en Roma.

Olmedo, P. (1997) Voices of our past: Using oral history to explore funds of knowledge within a Puerto Rican family. Anthropology and Education Quarterly, 28 (4), 550-73.

Poveda, D. (2001) La educación de las minorías étnicas desde el marco de las continuidadesdiscontinuidades familia-escuela. Gazeta de Antropología, 17, 17-31.

Ratner, C. (2011) Macro cultural psychology. A political philosophy of mind. New York, Oxford University Press. 
LA PRÁCTICA EDUCATIVA DESDE LA PERSPECTIVA DE LOS FONDOS DE CONOCIMIENTO E IDENTIDAD

Reyes, I. y Esteban-Guitart, M. (en prensa) Exploring multiple literacies from homes and communities: A cross-cultural comparative analysis, en HALl, K.; Cremin, T.; COMBer, B. y Moll, L. (eds.) International Handbook of Research on Children's Literacy, Learning and Culture. Maiden, Mass., Wiley-Blackwell.

Ríos-Aguilar, C. (2010) Measuring funds of knowledge: contributions to Latina/o students' academic and non-academic outcomes. Teachers College Record, 112 (8), 2209-2257.

Ríos-Aguilar, C.; Kiyama, J. M.; Gravitt, M. y Moll, L. (2011) Funds of knowledge for the poor and forms of capital for the rich? A capital approach to examining funds of knowledge. Theory and Research in Education, 9 (2), 163-184.

Rogoff, B. (1990) Apprenticeship in Thinking. Cognitive Development in Social Context. New York, Oxford University Press.

Salomon, G. (2001) Cogniciones distribuidas. Consideraciones psicológicas y educativas. Buenos Aires, Amorrortu.

SANDOVAL-TAYLOR, P. (2005) Home is Where the Heart Is: Planning a Funds of Knowledge Based Curriculum Module, en González, N.; Moll, L. y Amanti, C. (eds.) Funds of knowledge. Theorizing practices in households, communities, and classrooms. Mahwah, Lawrence Erlbaum Associates, 153-165.

Saubich, X. y Esteban-Guitart, M. (2011) Bringing funds of family knowledge to school. The living Morocco project. Multidisciplinary Journal of Educational Research, 1 (1), 79-103.

SCRIBNER, S. (1990) Reflections on a model. The Quarterly Newsletter of the Laboratory of Comparative Human Cognition, 12, 90-94.

Trumbull, E.; Rothstein-Fisch, C.; Greenfield, P. M. y Quiroz, B. (2001) Bridging cultures. Between home and school. A guide for teachers. Mahwah, Lawrence Erlbaum Associates.

VÉLEZ-IBÁÑEZ, C. (1983) Bonds of mutual trust: The cultural Systems of rotating credit associations among urban Mexicans and Chicanos. New Brunswick, NJ, Rutgers University Press.

- (2010) An impossible living in a transborder world. Culture, confianza and economy of Mexican-origin populations. Tucson, Az, Arizona University Press.

VÉLEZ-IBÁÑEZ, C. y GREENBERG, J. (1992) Formation and transformation of funds of knowledge among U. S. mexican households. Anthropology and Education Quarterly, 23 (4), 313335.

VILA, I. (1998) Familia, escuela y comunidad. Barcelona, Horsori Editorial.

VILA, I. y CASARES, R. (2009) Educación y sociedad. Una perspectiva sobre las relaciones entre la escuela y el entorno social. Barcelona, Horsori Editorial.

VyGOTKSI, L. S. (1962) Thought and language. Cambridge, MA, MIT Press.

- (1979) El desarrollo de los procesos psicológicos superiores. Barcelona, Ed. Grijalbo.

- (1997) Obras escogidas. Vol. V. Fundamentos de defectología. Madrid, Fundación Infancia y Aprendizaje/Visor.

WoOD, D.; BRuner, J. y Ross, G. (1976) The role of tutoring in problem solving. Journal of Child Psychology and Psychiatry, 17, 89-100.

ZIPIN, L. (2009) Dark funds of knowledge, deep funds of pedagogy: Exploring boundaries between lifeworlds and schools. Discourse: Studies in the Cultural Politics of Education, 30 (3), 317-331. 